\title{
The two faces of MycP1
}

Mycobacterium tuberculosis uses ESX1, a type VII secretion system, to deliver virulence factors into host cells. Because some of the proteins secreted by ESX1, such as ESAT6 (also known as EsxA), are important for virulence and also highly immunogenic, the system is transcriptionally regulated to maintain a balance between virulence and immunogenicity. Ohol et al. now reveal that ESX1 is further regulated by the subtilisin-like serine protease

$\mathrm{MycP} 1$, which is part of the ESX1 complex.
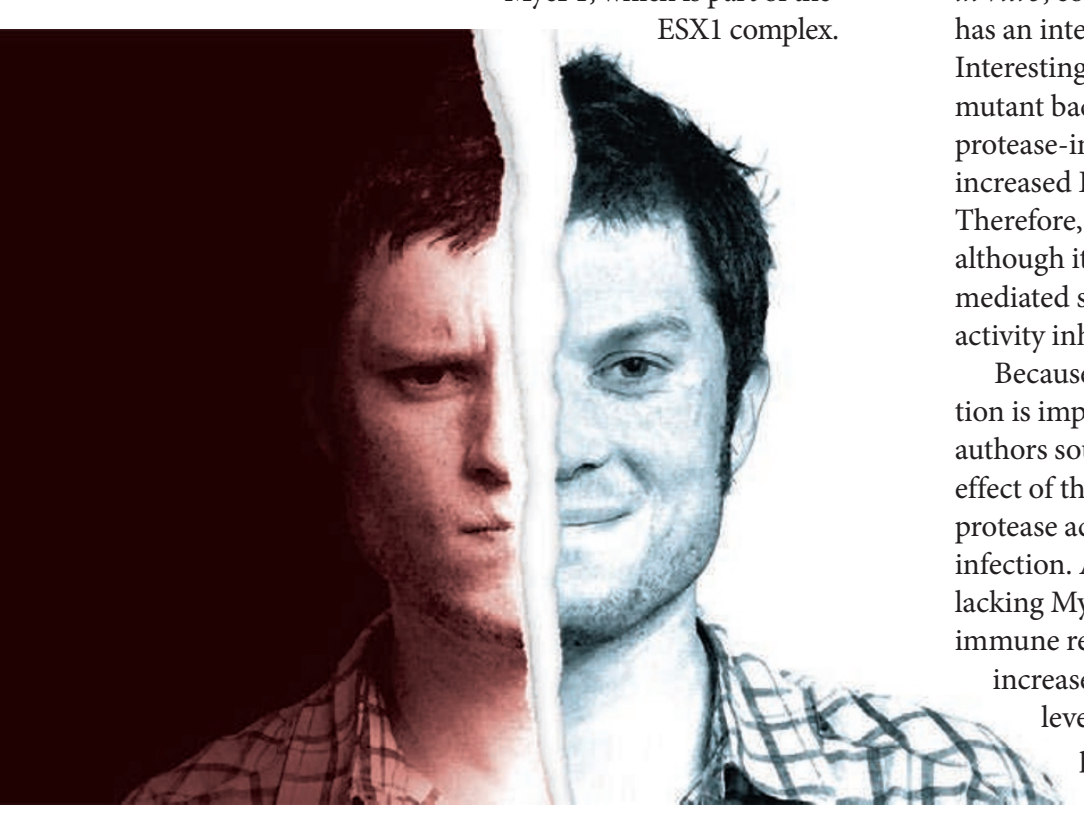

Clues that MycP1 might regulate ESX1 came from the observation that ESX1-secreted protein B (EspB) is cleaved by an unknown protease at its carboxyl terminus following secretion - the C terminus of EspB is required for interaction with and, consequently, secretion of ESAT6. Indeed, the authors showed that EspB is processed by $\mathrm{MycP} 1$ in vitro. Furthermore, loss of MycP1 abolished ESX1-mediated secretion in vitro, confirming that $\mathrm{MycP} 1$ has an integral role in this process. Interestingly, however, MycP1mutant bacteria reconstituted with protease-inactive $\mathrm{MycP} 1$ showed creased ESX1-mediated secretion. Therefore, $\mathrm{MycP} 1$ has a dual role: although it is required for ESX1mediated secretion, its protease ivity inhibits this process.

Because ESX1-mediated secreon is important for virulence, the authors sought to investigate the effect of the loss of MycP1 or $\mathrm{MycP} 1$ protease activity in macrophage infection. As expected, bacteria acking MycP1 did not elicit a strong mune response (for example, creased interferon- $\beta$ mRNA levels). However, bacteria with protease-inactive $\mathrm{MycP} 1$ triggered a more robust immune response than wild-type bacteria, which suggests that the loss of $\mathrm{MycP} 1$ protease activity induces enhanced innate immune responses by increasing ESX1-mediated secretion, in particular of immunogenic proteins such as ESAT6. These findings were also confirmed in vivo: mice infected with MycP1-mutant bacteria and bacteria carrying protease-inactive $\mathrm{MycP} 1$ showed higher survival rates than those infected with wild-type bacteria.

On the basis of their findings, the authors propose that $\mathrm{MycP} 1$ might be necessary for ESX1-mediated secretion because it is required for complex formation. However, MycP1 inhibits further ESX1-mediated secretion by cleaving full-length EspB (the protein that promotes the secretion of other substrates, such as ESAT6), thereby avoiding immune detection and ensuring bacterial survival.

Rachel David

ORIGINAL RESEARCH PAPER Ohol, Y. M. et al. Mycobacterium tuberculosis Myc $\mathrm{P} 1$ protease plays a dual role in regulation of ESX-1 secretion and virulence. Cell Host Microbe 7, 210-220 (2010)

FURTHER READING Abdallah, A. M. et al. Type VII secretion - mycobacteria show the way. Nature Rev. Microbiol. 5, 883-891 (2007) 